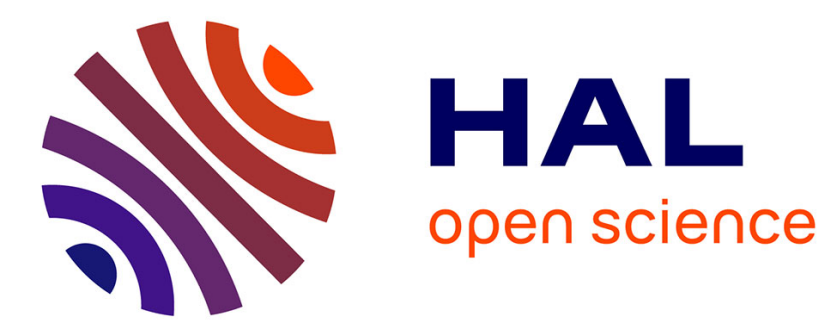

\title{
Finite elements and absorbing boundary conditions for scattering problems on a parallel distributed memory computer
}

\author{
Christian Vollaire, Laurent Nicolas, Alain Nicolas
}

\section{To cite this version:}

Christian Vollaire, Laurent Nicolas, Alain Nicolas. Finite elements and absorbing boundary conditions for scattering problems on a parallel distributed memory computer. IEEE Transactions on Magnetics, 1997, 33 (2 Part 2), pp.1448-1451. hal-00141613

\section{HAL Id: hal-00141613 \\ https://hal.science/hal-00141613}

Submitted on 23 Apr 2007

HAL is a multi-disciplinary open access archive for the deposit and dissemination of scientific research documents, whether they are published or not. The documents may come from teaching and research institutions in France or abroad, or from public or private research centers.
L'archive ouverte pluridisciplinaire HAL, est destinée au dépôt et à la diffusion de documents scientifiques de niveau recherche, publiés ou non, émanant des établissements d'enseignement et de recherche français ou étrangers, des laboratoires publics ou privés. 


\title{
Finite Elements and Absorbing Boundary Conditions for Scattering Problems on a Parallel Distributed Memory Computer
}

\author{
C. Vollaire, L. Nicolas, A. Nicolas \\ Ecole Centrale de Lyon - CEGELY - U.P.R.E.S.A C.N.R.S. 5005, B.P.163 - 69131 Ecully cedex - France
}

\begin{abstract}
An efficient finite element method is presented to compute time harmonic microwave field in three dimensional configurations. Nodal-based finite elements have been coupled with an absorbing boundary condition to solve open boundary problems. Modeling real devices is made possible using parallel computation. This paper describes first the time harmonic formulation with the absorbing boundary condition and the sequential code. A new algorithm is then proposed to implement this formulation on a cluster of workstations (10 DEC ALPHA 300X). Analysis of efficiency is performed using simple problems. The electromagnetic scattering of a plane wave by a perfect electric conductor cylinder is finally given as example.
\end{abstract}

\section{INTRODUCTION}

Nodal-based Finite Element (FE) method has been previously used to modelize microwave problems [1]. Absorbing Boundary Conditions ( $A B C$ ) have been introduced to simulate open boundary domains. The time harmonic formulation is written in terms of vector fields. Because nodal-based finite elements are used, a penalty term is added to the formulation in order to avoid spurious reflexions.

This code operates on scalar workstation: only simple problems can be modelized. For instance, a 3 wavelengths side cubic geometry, meshed with 10 nodes by wavelength, leads to 81000 complex unknows. Actually, only parallel computation enables to model real devices because it reduces the computation time and mainly arranges enough memory. For test considerations; we use a cluster of ten DEC ALPHA workstations (80 Mflops linpack, 64 Mo of RAM, 1 Go of swap per processor) linked by a FDDI ring. This distributed memory computer is a Multi Instruction - Multi Data streams (MIMD) type. Parallel Virtual Machine (PVM) software is used to pass messages between workstations.

The objective of this paper is to describe how the existing code has been modified in order to implement it on such a computer. Algorithms designed to operate on scalar calculators do not fit to parallel distributed memory computers because data are interdependant. It is not sufficient to add directives of messages passing in a program to allow good performances and algorithms have to be deeply modified. To implement this formulation on such a computer, we use an algorithm which distributes data over the different workstations. The developed code is a Single Program Multi Data type (SPMD). This allows to minimize messages passing which step is CPU consuming. Performances are then analyzed for simple problems. Speed-up, Amdahl's law and data volume switched are presented for the different steps of the program. The answer of a $6 \lambda$-length cylinder, modelized as a perfect electric conducting (p.e.c.), enlighted with an electromagnetic plane wave, is finally presented.

\section{FE FORMULATION COUPLED WITH ABC}

We are dealing with frequency domain open boundary electromagnetic field problems. According to Maxwell equations, the magnetic field $\mathbf{H}$ and the electric field $\mathbf{E}$ satisfy the vector wave equations. Following developements are made only for the $\mathbf{H}$ field formulation. All the steps can be applied to the $\mathbf{E}$ field formulation as well. The weak Galerkin formulation of the vector wave equation for $\mathbf{H}$ is given by:

$$
\begin{aligned}
& \int_{r}\left[\left(\nabla W \times \frac{1}{\varepsilon_{r}} \nabla \times \mathbf{H}\right)-W k_{0}^{2} \mu, \mathbf{H}\right] d v \\
& -\int_{r}\left[\mathbf{n} \times\left(W \frac{1}{\varepsilon_{r}} \nabla \times \mathbf{H}\right)\right] d s=0
\end{aligned}
$$

A penalty term is added to the formulation to avoid spurious reflexions [1]. It makes the field divergence free. $A B C$ are used to truncate the $3 \mathrm{D}$ finite element region. The open boundary is modeled using a second order 3D vector Enquist-Majda ABC [2]. Outer surfaces are then rectangles, which is less mesh-consuming for a large number of geometries:

$$
\mathbf{n} \times \nabla \times \mathbf{H} \cong g_{A B C}(\mathbf{H})=j k_{0} \mathbf{H}_{t}-\frac{j}{2 k_{0}} \nabla_{,}^{2} \mathbf{H}_{i}
$$

Finally, the formulation for scattering problems in term of total field is given by (3). Two types of surface are considered: external surface (Sext) and pec surface (Spec).

$$
\begin{aligned}
& \int\left[\left(\nabla W \times \frac{1}{\varepsilon_{r}} \nabla \times \mathbf{H}\right)+W k_{0}^{2} \mu_{r} \mathbf{H}\right] d v \\
& -\int_{r}[(\nabla W)(\nabla \cdot \mathbf{H})] d v+\int_{s e x t+S p e c} W \mathbf{n} \cdot \nabla \cdot \mathbf{H} d s \\
& -\int_{S e S t} W g_{A B C}(\mathbf{H}) d s=\int_{S e X t} W\left[g_{A B C}\left(\mathbf{H}_{i}\right)-\mathbf{n} \times \nabla \times \mathbf{H}_{i}\right] d s
\end{aligned}
$$

Where $\boldsymbol{H}$ is the total field and $\boldsymbol{H}_{i}$ the incident field 


\section{SEQuential Code}

Main steps of the existing code are:

1) Create the structure of the global matrix: symbolic assembling.

2) Assemble the global matrix: each elementary matrix (related to a volume finite element) is computed and results are then put in the global matrix.

3) Introduce $A B C$ on the external boundaries and Boundary Conditions ( $B C$ ) on conductors: only surface elements are seeked.

4) Introduce $B C$ on the symmetry planes.

5) Symmetrization of the matrix: the $\mathrm{ABC}$ step (\#3) results in an non-symmetric global matrix. The FE matrix is symmetrized by adding it to its transposed matrix. This approximative method has been shown to give good results

6) Solve the system of equations: because the matrix system is sparse iterative solvers have been implemented.

\section{PARALLEL FE ALGORITHM}

\section{A. Distributing the data}

We have chosen to duplicate the entire data file on each processor. Indeed, the program is a SPMD type performing a parallel reading. An other strategy would consist in storing no more than what is required for one processor at a time and in reading and updating data files during the processing. But this method would result in unacceptable $\mathrm{I} / \mathrm{O}$ overhead. A compromise is available in the case of distributed memory architectures [3]-\{5].

\section{B. Assembling in parallel}

The assembling step of the global matrix is performed by degree of freedom [6]. instead of by element as in the sequential code. It allows to assemble at once the three lines (three coordinates) corresponding to a node. Each element including the considerated node are seeked and contributions with the related nodes are computed. These three lines are then compressed by storing only non-zero values. Space is left in the compressed storage if the volume element includes a surface element. Same method is used to introduce $A B C$ and $\mathrm{BC}$ on conductors but only surface elements are seeked.

This method does not require the creation of the structure of the matrix. To solve a problem meshed with $\mathrm{N}$ nodes, if $\mathrm{W}$ workstations of the cluster are available, each workstation assembles a part of the system including N/W nodes. The load balancing is nearly perfect because of the constant bandwidth of the global matrix. The program is a SPMD type: no message passing is required. Hence, the speedup of the assembling stage is optimal (Fig. 4)

\section{BC on symmetry planes and conductors}

Messages passing are necessary to introduce the $\mathrm{BC}$ on conductors ( $\mathbf{E}$ field solving only) and on the symmetry planes because a global modification of the matrix system is required (due to the method used to introduce BC) (Fig. 1). On a symmetry plane the $H$ field verifies $(6 a)$ or $(6 b)$ :

On a symmetry plane:

$$
\mathbf{n} \cdot \mathbf{H}=0
$$

On an antisymmetry plane:

$\mathbf{n} \times \mathbf{H}=\mathbf{0}$

The speedup related to this step is very bad due to the large amout of message passing (Fig. 4). However, its effect on the global performances of the code is negligible: the length of this step is less than one percent ( $\mathbf{H}$ field solving) of the total computation duration (Fig. 2).
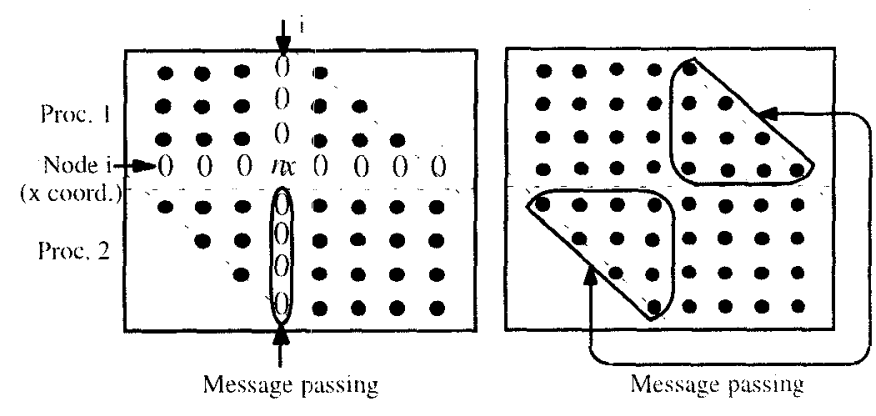

Fig. 1. Message passing for the symmetry planes (left) and for symmetrization (right) - Example with 2 processors.

\section{Symmetrizing the global matrix}

The introduction of $\mathrm{ABC}$ and penalty function results in a non-symmetric system matrix. This one is approximatly symmetrized by adding it to its transposed matrix. This operation needs a large amout of messages passing (Fig. 1). The speedup related to this operation is also poor (Fig. 4). But, once more, the time needed to perform this step is small compared to the total computation time (Fig. 2).

\section{E. Solving in parallel}

Quasi Minimum Residu (QMR) and Conjuguate Gradient (CG) with diagonal or incomplete Choleski preconditioner have been implemented [7], [8]. Because these methods are iterative, small-scale parallelism is used. Parallel matrix multiplication allows to compute the residual vector of each itcration: each processor computes a partial residual vector and broadcasts it to all the others (only non-zero values are sent). Then, each processor adds these partial residual vectors to obtain the final residu (SPMD). An other way to calculate the residual vector is to send each partial residual vector to one processor. This one computes the final residu and 
broadcasts it. This Master / Slave (M/S) method minimizes the number of communications.

The cost of communications in this step is penalizing, because small-scale parallelism is not adapted to distributed memory computer. Some others methods can be found in [9] but they are more memory space consuming.

\section{PARALlEL PERformanCES}

PARAGRAPH is used to analyse the performances of the code. For such a purpose, 2 small problems (scattering by a pec cylinder) have been modelized: the first one is meshed with 4000 nodes, leading to 12000 degrees of freedom, and the second one is meshed with 10000 nodes, leading to 30000 degrees of freedom.

\section{A. CPU time distribution}

CPU limes to solve both of these problems on 8 processors were, respectively, $236 \mathrm{~s}$ and $688 \mathrm{~s}$. Fig. 2 shows the CPU time distribution for the main steps of the code ( 8 CPU's). The solving step is the most CPU consuming because its speedup is very poor (Fig. 4).

Note that in a sequential computing, the assembling step would spend $60 \%$ of the CPU time and the solver only $35 \%$.

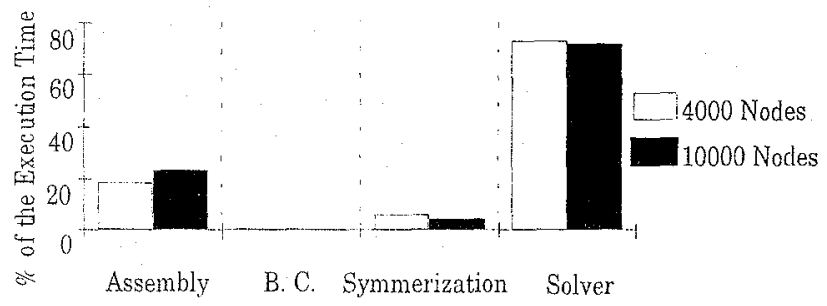

Fig. 2. CPU time distribution (8 CPU's)

\section{B. Analysis of a 4000 nodes problem}

For the problem meshed with 4000 nodes, Fig. 3 shows the state of the eight processors (busy, overhead or idle) and Table I shows the data quantity switches. This analysis has been done for both of the programming types of the solver. The M/S method is performed with the processor \#1 as Master. The idle time corresponds to synchronisation phases. The overhead time is introduced by the messages passing. From Fig. 3, the SPMD type appears more efficient. However, for large problems, it can imply a saturation of the communication network (Table I). In fact, SPMD type seems to be more adapted to a 'crossbar' network. The data quantity switches remain approximatively the same for both types of programming, but the number of messages decreases for the $\mathrm{M} / \mathrm{S}$ type. The load balancing is good for both programming types.
TABLE I

DATA SWITCHES (4000) NODES)

\begin{tabular}{ccccccc}
\hline \multirow{2}{*}{$\begin{array}{c}\text { processor } \\
\text { number }\end{array}$} & \multicolumn{2}{c}{$\begin{array}{c}\text { number of } \\
\text { messages }\end{array}$} & \multicolumn{2}{c}{ Mb received } & \multicolumn{2}{c}{ Mb sent } \\
& M/S & SPMD & M/S & SPMD & M/S & SPMD \\
\hline 1 & 3171 & 3171 & 99.2 & 99.2 & 64.3 & 81 \\
2 & 471 & 3171 & 93.2 & 95.7 & 16 & 106 \\
3 & 471 & 3171 & 93.3 & 96.2 & 15.8 & 102 \\
4 & 471 & 3171 & 93.28 & 95.8 & 16.3 & 105 \\
5 & 471 & 3171 & 93.31 & 97.1 & 15.8 & 96 \\
6 & 471 & 3171 & 93.2 & 97.3 & 14.7 & 94.6 \\
7 & 471 & 3171 & 93.24 & 97 & 15 & 95 \\
8 & 471 & 3171 & 93.40 & 97.3 & 13.7 & 94.7 \\
\hline
\end{tabular}

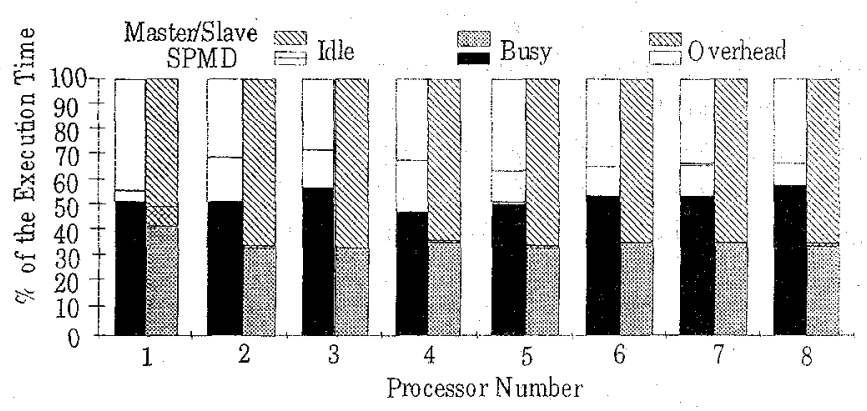

Fig. 3. Processors states for a 4000 nodes problem.

\section{Speedup versus number of processors}

For a given problem, the sequential ratio of the code remains constant but the cost of communications increases with the number of processors. Parallel ratio given by the Andahl's law increases slightly with the number of processors (Table II and Fig. 4).

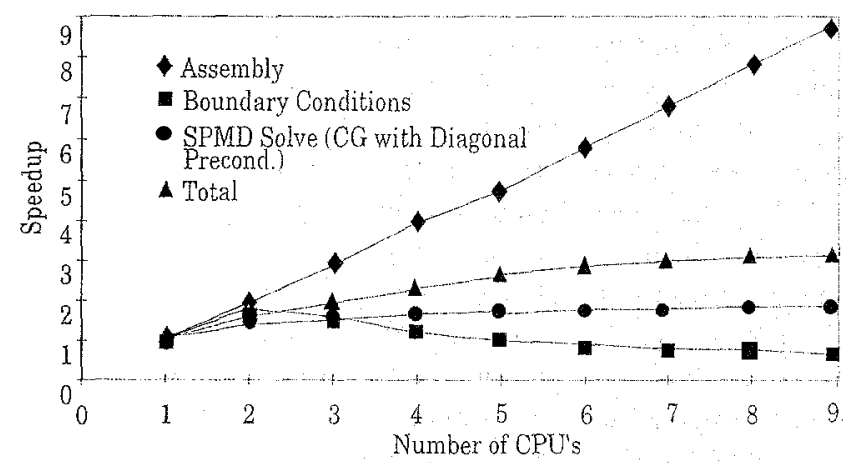

Fig. 4. Speedups for a 10000 nodes problem.

TABLE II

PROBLEM MESCHED WITH IOONO NODES

\begin{tabular}{ccccccccc}
\hline CPU's & 2 & 3 & 4 & 5 & 6 & 7 & 8 & 9 \\
Speedup & 1.5 & 1.9 & 2.2 & 2.5 & 2.8 & 3 & 3.17 & 3.21 \\
Amdahl's law & $66 \%$ & $71 \%$ & $72 \%$ & $75 \%$ & $76 \%$ & $77 \%$ & $78 \%$ & $77 \%$ \\
\hline
\end{tabular}




\section{Speedup versus number of nodes}

From Table III, it is obvious that the efficiency of the method increases with the size of the computed problem: this is due to the communication times witch become negligible compared to computation times.

When computing large problems, it becomes difficult to measure the code performances because these problems cannot be computed on only one processor (the speedup can not be calculated !). A theoretical variation of the CPU time on 1 processor depending on the number of mesh nodes $(n)$ is then used. This theoretical variation is calculated from the actual variation obtained for problems meshed with 10000 nodes at most, together with the knowledge that solving time varies as $n^{2}$ (Table IV).

TABLE III

RESULTS FOR $\times$ CPU'S

\begin{tabular}{cccc}
\hline $\begin{array}{c}\text { number of } \\
\text { nodes }\end{array}$ & total speedup & solving speedup & $\begin{array}{c}\text { Amdahl law } \\
\text { (total speedup) }\end{array}$ \\
\hline 3000 & 2.35 & 1.2 & $65 \%$ \\
4000 & 2.62 & 1.56 & $70 \%$ \\
10000 & 3.17 & 1.76 & $78 \%$ \\
15000 & 3.61 & 1.81 & $82.6 \%$ \\
\hline
\end{tabular}

TABLE IV

RESULTS FOR 8 CPU'S

(CPU TIME ON I PROCESSOR HAS BEEN ESTIMATED)

\begin{tabular}{ccc}
\hline number of nodes & total speedup & Amdahl law \\
\hline 22000 & 3.9 & $84.9 \%$ \\
$3800(0$ & 4.2 & $87 \%$ \\
52000 & 4.5 & $88.8 \%$ \\
65000 & 4.9 & $90 \%$ \\
\hline
\end{tabular}

\section{Modeling The SCATtering By A $6 \lambda$ CYlinder}

In this section, we present the example of a cylinder enlighted with an electromagnetic plane wave (Fig. 5). The length of the cylinder is $6 \lambda$, and the radius is $0.5 \lambda$. The frequency of the incident wave is $3 \mathrm{GHz}$. Problem has been modelized with 1 symmetry. To obtain accurate results, 10 nodes per wavelenght are used [1], leading to a mesh made of 50000 nodes. Note that the space memory available on the cluster of stations allows to solve problems meshed at most with 100000 nodes.

\section{CONCLUSION}

We have presented in this paper the implementation of an electromagnetic scattering code on a parallel memory computer, Parallel performances obtained when building the FE matrix are satisfying. On an other hand, the cost of communications in the solver is penalizing, because small- scale parallelism is not adapted to distributed memory computer.

The modeling of realistic problem is not possible on such a cluster because of the memory available. As example, the study of an $15 \mathrm{~m}$-length airplane, even simplified as a perfect electric conducting body, enlighted by a $3 \mathrm{GHz}$ plane wave, would lead immediately to a problem meshed with more than $10^{6}$ nodes.

On an other hand, this code is immediately implementable on a computer such as the CRAY T3D. This parallel distributed memory computer is a MIMD type. Given its characteristics ( 128 processors and 8 Go of memory), it should allow to arrange enough memory to modelize realistic devices.

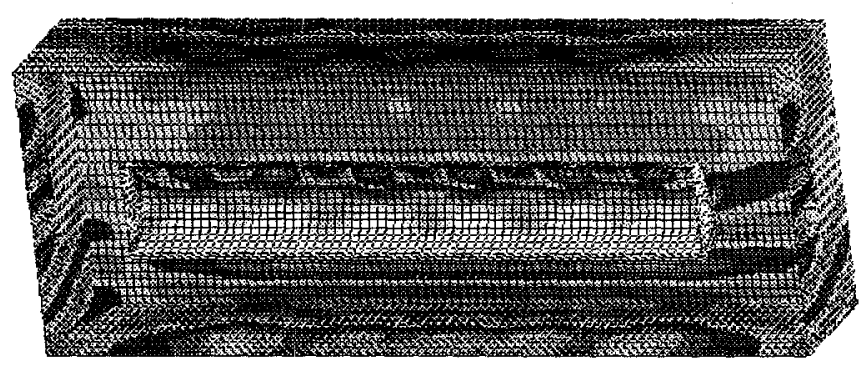

Fig. 5. P.E.C. cylinder meshed with $500(0)$ nodes Magnitude of the magnetic field.

\section{REFERENCES}

[1] L. Nicolas, K. A. Connor. S. J. Salon, B. G. Ruth, and L. F. Libelo. "Three dimensional FE analysis of high power microwave devices." IEEE Trans. Mag., no. 2, pp. 1642-1645, March 1993.

[2] J. L. Yaobi, L. Nicolas, and A. Nicolas, "Vector absorbing boundary conditions for nodal or mixed based finite elements," to be published in IEEE Trans. Mag., May 1996.

[3] G. J. Bendzsak, and T. W. Ma, "Parallel computation of 3-D electric and magnetic fields." IEEE Trans. Mag., vol. 27, no. 5. pp. 4205-4209. September 1991.

[4] S. Ratnajeevan, and H. Hoole, "Finite element electromagnetic ficld computation on the sequent symmetry 81 parallel computer," IELE Trums. Mags., vol. 26, no. 2. pp. 837-840, March 1990.

[5] M.L.Barton, "Three-dimentinal magnetic field computation on a distributed memory parallel processor," IEEE Trans. on Mag. vol. 26. no. 2, pp. 834-836. March 1990 .

[6] D. Zois "Parallel processing technigues for $\mathrm{FE}$ analysis: stiffnesses. loads and stresses evaluation," Comp. \& St. vol. 34, no. 3. pp. 355-374. 1990 .

[7] R. W. Freund, "Conjugate gradient type methods for linear systems with complex symmetric coefficient matrices." SIAM J. Stat. Commut., vol. 13, no. 1. January 1992.

[8] R. W. Freund, M. H. Gutknecht, and N. M. Nachtigal, "An implementation of a look-ahead Lanczos algorithm for non-hermitian matrices, Part I." Terf. Rep. 90.45. RIACS. NASA. Ames Researd Center, November 1990.

[9] R. F. Lucas, T. Blank, and J. J. Tiemann, "A parallel solution for large sparce systems of equations," IEEE Trans Comp. Aided Des., vol. 6. no. 6, pp. 981-991, November 1987. 